\title{
Products as Affective Modifiers of Identities
}

\author{
Daniel B. Shank ${ }^{1}$ \\ Rohan Lulham ${ }^{2}$ \\ Forthcoming in Sociological Perspectives
}

\begin{abstract}
Are salesclerks seen as better, more powerful, or more active when they drive Mustangs? What about entrepreneurs? What about driving a mid-sized car? Intuitively we have ideas about these, but much of the research on the affective nature of products is on purchasing, desires, and self-fulfilment. Drawing on symbolic interactionism, we argue that people's association with products has some basis in the impression management of their identity. For this to occur there must be some cultural consensus about the way that products modify identities. Drawing on affect control theory's methodology and equations, we measure the goodness, powerfulness, and activeness of several products, identities, and the associated product-modified identities to explore how products function as affective modifiers of identities. We find consistent effects across several types of technology products whereby products pull the modified identity in the direction of the products' affective qualities. Support is established for the affect control theory equations that predict how traits modify identities as also having utility for predicting how products modify identities. This suggests that the opening questions can be answered empirically by measuring cultural-specific sentiments of the identity and the product and by developing equations to predict the identity modification process.
\end{abstract}

Acknowledgement: The data collection was supported by seed funding from the Designing Out Crime research centre, University of Technology Sydney. We thank Neil MacKinnon for his comments on an earlier draft.

\footnotetext{
${ }^{1}$ University of Melbourne, Melbourne, Australia
}

\section{Corresponding Author:}

Daniel B. Shank, Melbourne School of Psychological Sciences, University of Melbourne, VIC 3010,

Australia

Email: daniel.shank@unimelb.edu.au

${ }^{2}$ University of Technology Sydney, Sydney, Australia 


\section{Products as Affective Modifiers of Identities}

Are salesclerks seen as better, more powerful, or more active when they drive Mustangs? What about entrepreneurs who drive Mustangs? What about salesclerks who drive mid-sized cars? Intuitively we have ideas about these, but much of the research on the affective nature of products is on purchasing, desires, and selffulfilment, not about people’s impressions. Drawing on symbolic interactionism, the current research explores how people's impressions of others' identity change when that identity is associated with a product. To answer these opening questions, we employ an established framework and method from affect control theory in which impressions for identities and products are conceptualized in terms of their affective meaning.

Products are ubiquitous in social life with some products being specific to particular social roles (sports clothing), whereas others accompany individuals across a range of social roles (mobile phones). Much of the empirical research on the affective nature of products has occurred in the domains of product design and consumer research where individual's initial purchasing, emotion and self-fulfilment has been a focus (Desmet and Hekkert 2009; Loken 2006). Less design and marketing research has taken a sociological perspective where the interpersonal role of products in everyday life is investigated (Reed 2002; Solomon 1983). Considering the potential impacts of products on how we define and present ourselves, research that develops a better understanding of the role of products in social life is an important interdisciplinary development. 
In this article we draw on premises in symbolic interactionism to develop an initial framework for conceiving and investigating the role of products in sociocultural contexts. Our framework is built on Lulham's (2013) articulation of how products could be conceptualized as modifiers within affect control theory, a formal theory of symbolic interactionism. Before detailing this framework, we briefly discuss the literature in the design and marketing fields examining experience, design and consumption of products. Then, drawing on symbolic interactionism, we argue that people associate with and display products with particular affective meaning in part to alter how others perceive their identity. We identify two core research questions: How are the impressions of identities modified through association with a product? And can trait equations from affect control theory predict impressions of identities modified through association with a product? The investigation of these research questions in this paper represents an initial empirical study of products as affective modifiers of identity, designed to provide preliminary validation of the framework. After reporting the findings of the study, we discuss the implications and directions for future research and theory development.

\section{LITERATURE AND THEORY}

Affective Experience of Products

People's affective experience of products and brands is increasingly a core area of research for informing product design and marketing (Desmet and Hekkert 2009; Loken 2006). In product design research a dominant theme is how products make people feel and how designers can better create products that provoke beneficial or hedonistic emotional experiences (Desmet and Hekkert 2007; Norman 2004a; 
Norman 2004b). It includes the development of various methods for assessing people’s emotional responses to products (Desmet 2012), and the development of frameworks for designing emotional experiences into products (Desmet and Hekkert 2007; Norman 2002). In parallel in the marketing and consumer psychology fields, the majority of research investigates how consumer purchasing decisions are influenced by the experienced emotions related to products or other contextual factors (i.e., store environment, brand meaning, sales approach) (Loken 2006). This consumer research often takes an individual psychological approach seeking to better understand consumer affective experience to promote purchasing behaviour.

Studying the interrelationships between self-concept, identities and products is also a key interest in consumer psychology (Levy 1959). Rosenberg (1979) argues that material possessions such as products can be viewed an extension of one's selfconcept, and therefore they can be chosen or displayed in order confirm or enhance one’s self view. People generally choose or identify with products and brands that are consistent with how they view themselves (Reed et al. 2012; Sirgy 1982). This includes not only how people view themselves generally (self-concept) (Rosenberg 1979) but also how they view themselves in particular social roles (identities), with research indicating identities have a greater influence on product choice than broader self-concept (Kleine et al. 1993; Reed 2002). Whereas people's view of themselves and products are operationalized using a variety of concepts (personality, values, status), measures of affective quality and meaning are increasingly common (Coleman and Williams 2013; Kleine et al. 1993). In this research that uses affective measures, the focus is often around exploring whether people appraise, buy and use products that have similar affective meanings to how they view themselves in social 
roles. Some recent research also suggests an important social function of products is to facilitate people having feelings appropriate to their ascribed identity in social situations (Coleman and Williams 2013).

\section{Symbolic Interactionism and Products}

Most past consumer psychology and design research has largely focused on the within-individual psychological processes related to people’s purchasing, interaction with and experience of products. The overwhelming emphasis is on how products make people feel as opposed to how people feel about products. Counter to this focus, Solomon (1983) suggested products also operate as social symbols that promote experience and behaviour in social contexts. Drawing on symbolic interactionism, Solomon (1983) considers products as impacting not only on how people view themselves, but also how others view them in social situations. Products communicate social meaning to others, altering how one's identity is viewed and the associated behaviours expected to be performed in social situations. This view builds on the classic symbolic interactionism tenant that "Social beings are things as definitely as physical things are social” (Mead 1932).

Symbolic interactionism further presumes that humans are social animals that identify consensual meaning so they can collectively "act toward things in terms of their meaning” (McCall 2006:12). While consensual meaning is needed to facilitate interaction, interaction also facilitates the development, internal representation and manipulation of symbolic meanings (Stryker and Vyran 2003:4). Products, like identities and behaviour, are a source of meaning in social contexts. Therefore, in the 
course of human interaction, people can use products as part of this meaning making process.

Specifically, people can choose, associate with and use products to influence how other people perceive them in particular roles. In the modern era, a consumerist culture has encouraged people to acquire and display products for purposes related to image and status, not simply utility (Featherstone 1990). Veblen (2007 [1899]) was one of the first to articulate this position, arguing that higher class individuals engage in conspicuous consumption and leisure to bolster other's impression of their class status. Goffman’s theory of self-presentation (1959) suggests a more general process of impression management in which people use material objects as props to enhance their performance in an identity-role, be it class-based or not. Product association, therefore, may have multiple social psychological purposes. On one hand, people might associate with a product primarily to confirm their self-image to themselves (Rosenberg 1979), but, on the other hand, they might display products primarily as part of an impression management strategy (Goffman 1959; Solomon 1983). While much of the previous research is around confirmation of self-concept, our primary interest here is how products change people’s impressions of specific identity-roles. Essential to individuals' situational meaning making and interaction is their identity within a situation (Stryker 1980). People choose identities based on a number of factors including aspects of their self-concept (MacKinnon and Heise 2010), the salience of an identity (Stryker 1980), and the type of identity (Burke and Stets 2009). As people's identities and the products they associate with are subject to the same symbolic meaning-making and impression management processes, our central 
research question focuses on their interrelation. How are the impressions of identities modified through the association with a product?

\section{Affect Control Theory}

To specifically address our research question, we draw on affect control theory (ACT), a theory of symbolic interactionism (MacKinnon 1994) that specifies a process of identity modification (Averett and Heise 1987; Heise and Thomas 1989; Smith et al. 2001). Affect control theory (Heise 1979; Heise 2007; Robinson and Smith-Lovin 2006) is a theory of social interaction that asserts that our desire to maintain affective meanings about ourselves and the world around us is central to explaining and understanding how we feel, what we do, and the emotions we feel and communicate in situations. It is a formal theory that articulates a framework for the investigation of social interaction that includes identities, behaviours, emotions, traits, and settings. For a given language-culture, the affective qualities of each of these elements are assessed in isolation and in the context of events using standard semantic differential scales that measure dimensions related to the goodness, powerfulness and liveliness of the concept. This data is then used to model how the affective sentiments of identities such as entrepreneurs or salesclerks relate to ongoing impressions, behaviours, and emotions within interpersonal interaction.

Most relevant to our current research is the measurement of affective meaning and the trait modifier equations. Affective meaning is assessed in ACT on scales measuring the dimensions of Evaluation (bad to good), Potency (powerless to powerful) and Activity (inactive to active). These EPA scales, as they are often called, 
emanate from the pioneering work of Osgood and colleagues (Osgood et al. 1957; Osgood et al. 1975), who found compelling evidence that these three dimensions differentiated affective meaning for a range of concepts across cultures. Scholl's (2013) more recent review reiterates Osgood's findings and showed these three dimensions serve as a primary foundation in assessing human communication and interaction across a range of different disciplines. Notably for this research, these dimensions have been shown to reliably measure a range of linguistic concepts including objects and products (Shank 2010).

The aspect of ACT most important to this current study is the development of trait modifier equations that specify how traits (e.g., Sadistic, Obstinate) and emotions (e.g., Angry, Worried) can modify an identity (e.g., Doctor, Salesclerk). The traits and emotions were measured on EPA scales, the identities were measured on EPA scales, and then a selection of identities modified by traits and emotions were measured on EPA scales (e.g., Sadistic Doctor). Regressing the latter on the former, researchers first were able to develop the equations for trait-modification only (Averett and Heise 1987) and later to develop equations that allowed for either emotion or trait modification of identities (Heise and Thomas 1989). Therefore, these equations represent how people generally form an impression of someone based on their identity and the trait or emotion. The equations are,

$$
\begin{aligned}
& \mathrm{C}_{\mathrm{e}}=-.32+.69 \mathrm{M}_{\mathrm{e}}-.36 \mathrm{M}_{\mathrm{p}}+.47 \mathrm{U}_{\mathrm{e}}+.01 \mathrm{U}_{\mathrm{p}}-.07 \mathrm{U}_{\mathrm{a}}+.12 \mathrm{M}_{\mathrm{e}} \mathrm{U}_{\mathrm{e}} \\
& \mathrm{C}_{\mathrm{p}}=-.18-.18 \mathrm{M}_{\mathrm{e}}+.65 \mathrm{M}_{\mathrm{p}}+.01 \mathrm{M}_{\mathrm{a}}-.01 \mathrm{U}_{\mathrm{e}}+.59 \mathrm{U}_{\mathrm{p}}+.05 \mathrm{U}_{\mathrm{a}} \\
& \mathrm{C}_{\mathrm{a}}=-.11-.04 \mathrm{M}_{\mathrm{e}}+.07 \mathrm{M}_{\mathrm{p}}+.53 \mathrm{M}_{\mathrm{a}}-.02 \mathrm{U}_{\mathrm{e}}-.02 \mathrm{U}_{\mathrm{p}}+.64 \mathrm{U}_{\mathrm{a}}
\end{aligned}
$$


where $\mathrm{C}$ referring to the combined identity, $\mathrm{U}$ the unmodified identity and $\mathrm{M}$ the trait or emotion modifier, and the subscripts e, p, and a refer to evaluation, potency, and activity, respectively.

Using data collected on unmodified identities and modifiers, the trait equations can predict people's perception of modified identities. For example, affect control theory data collection indicates that a doctor in general is perceived as quite good (1.90), slightly powerful (.069) and neither active nor inactive (0.05) and the trait Sadistic is viewed as quite bad (-1.77), neither powerful nor weak $(-0.04)$, and slightly active $(0.76)^{1}$. Plugging those values into the trait equations predict that people will view a Sadistic Doctor as somewhat bad (-1.03), slightly powerful (0.51), and neither active nor inactive (0.34) based on the component values.

Emotions and traits are characteristics of individuals within situations, and therefore clearly related to the identity of the individual. It is no surprise that a sadistic doctor or an angry manager is perceived and treated differently than a doctor or manager without that respective characteristic. Drawing on the notion of modifiers within affect control theory, Lulham (2013) was the first to propose that products could similarly alter people’s fundamental sentiments for identities in social situations. He argued that rather than considering products as 'actors' in social events, in many situations the influence of products may be better conceived as modifiers of people’s identities. In the current research Lulham’s (2013) proposition is elaborated and explicitly tested. Do products associated with identities substantially modify those identities in a similar way to emotions and traits?

As we argued previously, the symbolic meaning of products should be an important aspect of impression management. Unlike trait and emotion characteristics, 
people are more volitional in determining what products to own, display, or be associated with, creating a particularly interesting dynamic. No previous research has investigated products as identity modifiers within the ACT framework and therefore we propose a second research question. Can the ACT trait modifier equations predict identities modified by association with products?

\section{AN EMPIRICAL TEST}

To examine our research questions we conducted an empirical test, collecting data on a select number of identities, products, and the product-modified identities. To create the modified identities six identities were crossed with twelve products that varied in type, status and whether branded or generic. Identities were selected from the business/economic domain as work is an institution with meaningful and stable identities (MacKinnon and Heise 2010). Previous affect control theory research on business and work identities also supports the theory’s utility in this context (Moore and Robinson 2006; Schneider 2002). Furthermore, the six identities were selected to cover a range of EPA values. Together the three EPA dimensions constitute a three dimensional space where any concept can be located using a coordinate system with the E, P, and A values. Different parts of EPA space are associated with different affective meanings depending on the value for each of the dimensions. We selected six identities (E, P, A) - Manager (0.91, 1.92, 1.54), Unemployed Person (-1.49, 2.37, -1.67), Salesclerk (0.67, -0.13, 0.52), Retiree (1.31, -0.32, -1.52), Entrepreneur $(1.44,1.82,1.78)$, and Scrooge $(-2.28,-0.17,-0.86)$ - to cover different regions of EPA space ${ }^{2}$. 
For products, we chose the domain of modern, well-known technology products as these achieve cultural consensus in EPA space (Shank 2010). Three particular concerns about the impact of product characteristics on modification processes were explored: the influence of the type of technology product (car verses phone), the status of the technology product (sports car verses mid-sized car) and the specific brand-name or generic version of the technology product (iPhone verses smart phone). These are all particularly important concerns for our design to incorporate, in order to understand whether these factors influence the impressions of people associated with products beyond their affective meanings. First, because different types of products serve different functions, they could exert different types of influence on people's identities. Second, high status products are high status because people agree they have more worth, and potentially because they have superior design or features (Zeithaml 1988) or because they display wealth or highclass tastes (Goffman 1951; Veblen 2007 [1899]). Therefore, status should be related to evaluation and potentially potency of the modified identity. Third, because companies spend millions to develop an image for their brand, differentiating it from other brands and generic versions of the same product, branded products may also have additional influences on affective impressions of product-modified identities (Aaker 1997).

To enable us to begin to address these concerns, for comparison we elected to examine three product types (automobiles, phones, and personal computers), including a medium and high status generic product term and two brand-name high status products. The final list included twelve products: Mid-Sized Car, Sports Car, Ferrari, Mustang, Mobile Phone, Smart Phone, Samsung Galaxy Phone, Apple 
iPhone, Laptop, Tablet Computer, Microsoft Surface, and Apple iPad. We crossed all six identities with all twelve products using the preposition "with,” e.g., Manager with a Tablet Computer, to create 72 product-modified identities.

\section{Methods}

We created an online survey in Qualtrics to obtain sentiment ratings of one of three sets of 50 concepts plus demographic questions. Each set included some of the unmodified identities, products, product-modified identities, additional products including for comparison only, and other concepts not used for this project. The concept sets were designed so that the product-modified identity (Manager with an Apple iPhone) was rated by a different group of participants than rated its components: the unmodified identity (Manager) and the product (Apple iPhone).

The evaluation, potency, and activity scales ranged from 'good, nice to bad, awful,' 'powerless, weak to powerful, strong,' and 'slow, quiet, inactive to fast, noisy, active', respectively (Heise 2010). The ordering and orientation of the scales were randomly presented per participant. Participants were allowed to indicate “Skip/Don’t Know" for any concept they did not wish to rate. Methodological validation of using semantic differentials to measure cultural meaning is provided by Heise (2010) and Osgood and colleagues (Osgood et al. 1957; Osgood et al. 1975).

\section{Participants}

We recruited US participants from the crowdsourcing marketplace Amazon Mechanical Turk (mturk.com) where participants could voluntarily complete our 
questionnaire for a small financial compensation. Whereas recruiting from Mechanical Turk is less common in sociology (Shank 2015), it has been found across the behavioural sciences to produce data for surveys and experiments that is at least as high quality as other data collection methods (Buhrmester et al. 2011; Mason and Suri 2012). Participants who have signed up for Mechanical Turk can self-select from the tasks that are posted, based on the time, pay, or interest.

Incomplete surveys were initially included in our data if at least 15 concepts were rated. Of the 254 participants initially included, 1 was eliminated due to skipping $40 \%$ of the concepts and 3 were eliminated due to polarities less than 0.6 (Heise 2010), meaning that they used excessive neutral or near neutral ratings. The remaining 249 participants included 130 women and 119 men, with a mean age of 33.7 (18 to 79). The participants live in 44 states and 1 territory with 88 from the West, 77 from the South, 49 from the Midwest, and 35 from the Northeast. Most identified as white (190; 72.5\%), Asian (28; 10.7\%), or Black (13; 5.0\%), and the majority (226; 86.3\%) had at least some college education. Our sample reflects the Mechanical Turk participant population which overall is similar to the US population, but with a skewed towards more women, younger, higher education, and more liberal attitudes (Berinsky et al. 2012; Mason and Suri 2012; Paolacci et al. 2010).

For each gender we obtained 34-44 ratings for all the concepts except Scrooge with a Microsoft Surface which received only 32 ratings from men and 27 from women. Therefore, the average ratings for these concepts are likely to provide a reliable indication of their affective meanings within US culture (Heise 2010; Osgood et al. 1957). Because our interest is in cultural meaning and impressions, the unit of analysis for all of the results is not the person, but the mean rating on each affective 
dimension (evaluation, potency, and activity) representing the general sentiments of a concept.

\section{Concept Ratings and Validity}

The unmodified identities (Table 1) received similar ratings compared to previous studies (Francis and Heise 2006) with the exception of Unemployed Person which is less negative than in previous studies ${ }^{3}$. We suspect this is due to impact of the 2008-2009 economic recession on individual's employment status being perceived as more contingent on societal and economic factors. A hierarchical logistic regression model predicting gender from ratings indicates no significant effect of identity EPA ratings $\left(\chi^{2}(\mathrm{df}=3, \mathrm{~N}=144)=4.35, \mathrm{p}=.23\right)$, but a significant and substantial effect of EPA product ratings, $\left(x^{2}(d f=6, N=144)=34.92, p \leq .001\right)$. Individual item t-tests (Table 1) show women have significantly higher ratings on at least one dimension (evaluation, potency, or activity) for a Mid-Sized Car, Smart Phone, Blackberry Phone, Apple iPad, and Amazon Kindle. Therefore, we control for gender in our analyses.

The product ratings (Table 1) displayed face and convergent validity with Sports Car and the specific sports cars were all seen as broadly similar in goodness, potency, and activity. Mid-sized Cars were much closer to being affectively neutral on all dimensions. For phones, Smart Phones were perceived as better, more powerful, and livelier than Mobile Phones. The specific brands tended to not be as highly regarded as the generic smart phone concept, but they had an affective ordering based on reputational status with the iPhone and Samsung Galaxy on top, followed by the Droid and Lumina, with Blackberries being dated enough to only 
receive near neutral sentiments. For computers, the more recent Tablet Computers are not yet as good, powerful and active as Laptops. The specific tablet computer brands are similar to the generic term, save Microsoft Surface which is lower on all three dimensions. Finally, Amazon Kindle has a noticeable difference in activity from other tablets, possibly from the design and early marketing of it as primarily an e-book reader.

Results

Research Question 1: How are the impressions of identities modified through association with a product?

To address our first research question, we first plot and describe the relationship for each observed product-modified identity to its components: the unmodified identity and the product. We then conduct a hierarchical linear regression predicting the 72 product-modified identities from the EPA values for the relevant identities and products, followed by variables for gender and other product characteristics.

Due to space limitations, we only graphically present the results for women however all results for men and women are in Appendix Table A. The patterns for men and woman are similar and lead to the same general conclusions, so our discursive discussion is applicable across genders. Figures 1-6 present the female data by identity: Entrepreneur, Manager, Salesclerk, Retiree, Unemployed Person, and 
Scrooge respectively, however the results are clearest when discussed by affective dimension.

Evaluation of a modified identity is quite similar to an unmodified identity across identities and products. Evaluation is slightly lower for Retirees modified with a product in spite of the products having higher evaluation. Only Scrooges, who unmodified are normally quite bad, are viewed as slightly bad when modified with a product.

For powerful identities, such as Entrepreneurs, Managers, and Scrooges, potent products appear to have little effect, however closer inspection suggests that when the product and unmodified identity have similar levels of potency, the modified identity follows the potency of the product as much or more than it does the unmodified identity. For the relatively powerless identities, such as Salesclerks, Retirees, and Unemployed People, the potency from the products increases the potency of the identity.

For activity, a similar pattern emerges. The activity of a number of the products is lower than the activity of Entrepreneurs, so when modified by the product people view Entrepreneurs' activity dropping to the level of the product. This can also be seen to a lesser degree for Managers and Salesclerks, whereas products increased the perceived liveliness of Retirees, Unemployed People, or Scrooges. For activity, and to a lesser extend potency, there is also a 'sports car effect', whereby a Sports Car, Mustang, or Ferrari which are each perceived as extremely active and potent, pull all identities toward their level of activity and power.

We conducted three hierarchical linear regressions, predicting the evaluation, potency or activity of the product-modified identity from the EPA ratings for the 
respective component identities and products. In each of the hierarchical linear regressions, the first model includes the unmodified identity’s evaluation, potency, and activity, with the second model adding the product's evaluation, potency, and activity. Our expectation is Model 2 to greatly improve the $\mathrm{R}^{2}$ over Model 1 . In Model 3 we add gender ${ }^{4}$ and in Model 4 we add product characteristics: branded versus generic, medium versus high status, and type (car, computer, or phone).

For predicting evaluation (Table 2), unmodified identities explain over $90 \%$ of the variance, with the evaluation of the identity being the largest coefficient (Model 1). Adding, product EPA significantly increases the $\mathrm{R}^{2}$, but only explains $1.3 \%$ more variance. Counter to our expectations, evaluation of the product is originally only marginally significant (Model 2), until gender is controlled for (Model 3; but see Model 4). While potentially interesting, the minimal impact in predicting the productmodified identities' evaluation based on the addition of a consumer product could be due to the unmodified identities explaining such a large portion of the variance or because many of the products chosen have similarly high evaluations.

For potency (Table 3), identity explains $68.4 \%$ of the variance and increases to 87.5\% when product ratings are included (Models 1 and 2). Potency of the unmodified identity and product are the primary contributors. For activity (Table 4), the unmodified identity explains $41.5 \%$ of the modified identities’ ratings, and product ratings more than double the amount of variance explained up to $88.2 \%$ (Models 1 and 2). Notably, as well as high coefficients for both the activity of unmodified identity and product, the potency and evaluation of the product contribute sizably to the modified identity’s activity level. 
Adding gender to the models (Tables 2-4: Model 3) indicated that women’s evaluation and activity rating for product-modified identities is lower than men's. This could be due to difference in initial product ratings for the products we selected or it could be actual differences in the amalgamation process. Thomas and Heise (1995) found no substantial gender differences in the amalgamation process for emotions and traits, so a different amalgamation process by gender for products would be an unexpected and interesting finding. As the products presented here did not cover the entire EPA space leading to increased correlations among the affective dimensions, this analysis can only be viewed as suggestive of gender effects. More clear than the gender effects were the overall lack of any differences for product attributes (Tables 2-3: Model 4), except that cars increase the liveliness of one's identity (Table 4: Model 4). Note that the lack of a status difference should be interpreted cautiously as we did not include any low status products, and received a range of ratings on our medium status products.

Both the discursive and statistical results indicate that how products modify identities is largely related to the affective qualities of the product - especially for potency and activity. Gender was related to this process, possibly due to affective differences in products by gender. After accounting for the influence of identity and product affective meaning, whether the product is of a specific or generic brand (e.g., Ferrari vs. Sports Car), of high or medium status (e.g., Midsize Car vs. Sports Car) or of a particular technology domain (e.g., computers, phones, cars), did not contribute to systematic differences in how products modified identities in our study. 
Research Question 2: Can the ACT trait modifier equations predict identities modified by association with a product?

To address our second research question, we input the EPA values for the relevant identities and products into the ACT trait equations to derived predicted values for each of the 72 product-modified identities (displayed in Figures 1-6). We are then able to compare visually and assess quantitatively the relationship between the predicted values derived using the ACT trait equations and the observed mean values for the 72 product-modified identities.

Across the identities, the evaluation predictions of modified identities were extremely similar to the observed, with people viewing Retirees with products as slightly worse than predicted. The equations slightly overpredicted potency and activity for high potency and activity identities, Entrepreneurs and Managers, with products. For Retirees, which are lower in both, the equations underpredicted the modified identities. For Unemployed People, which are lower in both, and Salesclerks and Scrooges, which are near neutral in both, the predictions were fairly accurate. Overall, the lines for predicted and observed modified identities showed similar patterns.

To confirm our discursive findings, we used $\mathrm{R}^{2}$ statistics to examine how much of the variation in the observed values for evaluation, potency and activity of the modified identities is explained by the values derived from the ACT trait equations ${ }^{5}$. Due to the gender differences found in the prior analyses, we calculated $\mathrm{R}^{2}$ for each gender separately. For evaluation, $\mathrm{R}^{2}$ for men was .82 and for women .87; for potency .84 for men and .83 for women; and for activity .81 for men and .78 for 
women. In other words the affect control theory trait equations, which were constructed based on emotions and traits as modifiers, predict the variation in identities modified with consumer products very accurately in our sample for both men and women. Notably, they account for nearly as much variation as the regression models which use the raw data to find the best solution. This is an encouraging finding, suggesting that trait equations have value for understanding consumer products as modifiers of identities.

\section{DISCUSSION}

Research in symbolic interactionism and the larger field of sociology indicate that one’s roles and identities establish culturally normative and affective expectations, scripts, and feeling rules that guide people's social experiences. Research in consumer psychology, marketing, and design indicate that consumer products evoke culturally normative affective meanings that relate to people's purchasing decisions, expectations, and self-sentiments. Our research combines these perspectives to suggest that the association with products within interpersonal interaction systematically modifies the sentiments of identities. The empirical findings suggest that people's impressions of product-modified identities come from the stable affective meanings of both the product and the unmodified identity. The evaluation dimension was the only one which showed mixed results, with product evaluation not displaying a clear pattern in its influence on identities. Possibly, this could be explained by the products' lack of variation on this dimension. We explored several product characteristics: different domains (e.g., phones, cars, computers), different 
statuses, and both generic and different brand-specific terms. The sentiments of the product-modified identity were consistently related to the affective meaning of the product and unmodified identity, not to these different product characteristics. From our data, people appear to use a somewhat consistent identity and product amalgamation process based on affect to determine the sentiments of the modified identity, regardless of the product characteristics.

What is the nature of this systematic amalgamation? No literature or theory we know of directly considers how products systematically alter how people’s identities are perceived. In this study we drew on affect control theory to argue that within interpersonal interaction a product's affective meaning may systematically modify an identity's goodness, power, or liveliness. This argument built on the trait equations in ACT for modelling identities modified by emotions and traits. We suggest the process of identity modification could extend into products, and potentially into other types of ownership, objects, or props (Goffman 1959). In this study these trait identity modification equations were promising in their ability to forecast the affective qualities of product-modified identities. This was the case notwithstanding that products were outside of the implicit scope of these trait equations. We believe this study provides preliminary evidence that affect control theory is an appropriate basis for modelling how products change the way people are perceived in social situations. In so doing, this research confirms Lulham’s (2013) initial proposition and further provides a basis for building a framework around ACT for explaining people's use of products to modify impressions of themselves when occupying particular identities.

Conclusions from this study, while promising, are not definitive due to some limitations of our data and methods. We made several trade-offs in design. This 
included choosing three product characteristics (domains, brands, status), and one domain of identities, and a full crossover of identities and products, instead of breadth of identities and products. For identities, there is strong evidence from affect control theory that impression processes are consistent across identities from different social institutions (MacKinnon and Heise 2010). Therefore, we would assume there is not a unique process that occurs for the work and economic identities that we selected, but the generalizability will need to be empirically verified in future research.

Our data were limited in their representation of the EPA space. The analyses in this paper, while compelling, are not comprehensive. Without full coverage of the EPA space it was not possible to develop the same quantitative models as created by Thomas and Heise. However, this research is required to create greater certainty about our findings and we intend to conduct this future research with a breath of products as we discuss next. While this broader study is needed, the value of the approach taken in this study - focusing on a discrete number of product and identities - is evident. It enabled more detailed and descriptive analysis for the initial tests of our research questions.

For a breadth of products, we recommend future research considers a widerange of types of products and a wide range of product sentiments. In this study, notably, our products were all considered good, potent, and active, although to different degrees. Companies desire their products be viewed in this way, so finding commercially produced consumer products that people perceive as awful, impotent, or dull could be difficult. However, Shank (2010) surveyed the EPA of technology products, finding outdated products tended to be negative in all three dimensions and weapon products to be negative in evaluation, while positive in potency and activity. 
Future research would need to establish whether products exist that represent all octants of EPA space (e.g., positive and negative for each dimension). Whereas there is considerable variance in all three EPA dimensions, it may be difficult to find a number of concepts for a particular combination (e.g., Heise and Thomas 1989). Collecting data on products, and modified and unmodified identities distributed across EPA space would enable the development of modifier equations for products in a similar way as was done for emotions and traits (Averett and Heise 1987; Heise and Thomas 1989).

\section{Conclusion}

In this research, salesclerks are seen as more powerful and more active when they drive Mustangs, but no better or worse. Yet entrepreneurs who are normally seen as quite good, potent, and lively are seen as slightly worse and less powerful when they drive Mustangs. In contrast, driving a mid-sized car decreases the goodness, power, and liveliness of entrepreneurs, whereas it increases the power and decreases the liveliness of salesclerks. Some of these findings are intuitive, but others are not. By uncovering specific findings like these we are prompted to ask if there is systematic influence of products on identity impressions. We have suggested that there is and it can be understood through affect control theory operationalising premises from symbolic interactionism. This provides exciting possibilities for both sociology and consumer science. With additional research it would be possible to include products as identify modifiers within the affect control theory framework enabling the simulation of how products change the broader dynamics in social situations. 
Practically for designers and marketers this research also suggests the importance of considering products as social symbols that change meanings in everyday life.

Products are more than functional items that can make us feel good. Products can change how we are viewed and how we view others, and as such, could be more fundamental than previously thought to people's management of impressions of self in social life. 


\section{REFERENCES}

Aaker, Jennifer L. 1997. "Dimensions of brand personality." Journal of marketing research:347-356.

Averett, Christine P. and David R. Heise. 1987. "Modified Social Identities: Amalgamations, Attributions, and Emotions." Journal of Mathematical Sociology 13:103-132.

Berinsky, Adam J., Gregory A. Huber and Gabriel S. Lenz. 2012. "Evaluating Online Labor Markets for Experimental Research: Amazon.com's Mechanical Turk." Political Analysis 20(3):351-368.

Buhrmester, Michael, Tracy Kwang and Samuel D. Gosling. 2011. "Amazon's Mechanical Turk A New Source of Inexpensive, Yet High-Quality, Data?" Perspectives on Psychological Science 6(1):3-5.

Burke, Peter J. and Jan E. Stets. 2009. Identity Theory. Oxford: Oxford University. Coleman, Nicole Verrochi and Patti Williams. 2013. "Feeling Like My Self: Emotion Profiles and Social Identity." Journal of Consumer research 40(2):203-222.

Desmet, Pierre and P Hekkert. 2009. "Special issue editorial: Design \& emotion." International Journal of Design 3(2):1-6.

Desmet, Pieter 2012. "Faces of product pleasure: 25 positive emotions in humanproduct interactions." International Journal of Design 6(2):1-29.

Desmet, Pieter and Paul Hekkert. 2007. "Framework of product experience." International Journal of Design 1(1):57-66.

Featherstone, Mike. 1990. "Perspectives on Consumer Culture." Sociology 24(1):522. 
Francis, Clare and David R. Heise. 2006. "Mean Affective Ratings of 1,500 Concepts by Indiana University Undergraduates in 2002-3." Program Interact $<$ http://www.indiana.edu/ socpsy/ACT/interact/JavaInteract.html $>$.

Goffman, Erving. 1951. "Symbols of Class Status." The British Journal of Sociology 2(4):294-304.

—. 1959. The Presentation of Self in Everyday Life. Garden City, NY: Doubleday. Heise, David R. 1979. Understanding Events: Affect and the Construction of Social Action. New York: Cambridge University Press.

—. 2007. Expressive Order: Confirming Sentiments in Social Actions. New York: Springer.

—. 2010. Surveying Cultures: Discovering Shared Conceptions and Sentiments: Wiley.

Heise, David R. and Lisa Thomas. 1989. "Predicting Impressions Created by Combinations of Emotion and Social Identity." Social Psychology Quarterly 52(2):141-148.

Kleine, Robert E., Susan Schultz Kleine and Jerome B. Kernan. 1993. "Mundane consumption and the self: a social-identity perspective." Journal of Consumer Psychology 2(3):209-235.

Levy, Sidney J. 1959. "Symbols for sale." Harvard business review 37(4):117-124.

Loken, Barbara. 2006. "Consumer psychology: categorization, inferences, affect, and persuasion." Annu. Rev. Psychol. 57:453-485.

Lulham, Rohan. 2013. "A theory of affective experience." in 19th International Conference on Engineering Design (ICED13). Seoul, Korea. 
MacKinnon, Neil J and David R Heise. 2010. Self, Identity, and Social Institutions: Palgrave Macmillan.

MacKinnon, Neil J. 1994. Symbolic Interactionism as Affect Control. New York: State University of New York.

Mason, Winter and Siddharth Suri. 2012. "Conducting behavioral research on Amazon’s Mechanical Turk." Behavior Research Methods 44(1):1-23.

McCall, George J. 2006. "Symbolic Interaction." in Contemporary Social Psychological Theories, edited by P. J. Burke. Stanford: Stanford University Press.

Mead, George Herbert. 1932. The Philosophy of the Present. Amherst, New York: Prometheus Books.

Moore, Christopher D and Dawn T Robinson. 2006. "Selective identity preferences: Choosing from among alternative occupational identities." Advances in Group Processes 23:253-281.

Norman, Don. 2002. "Emotion \& design: attractive things work better." interactions $9(4): 36-42$.

Norman, Donald A. 2004a. Emotional design: Why we love (or hate) everyday things: Basic books.

—. 2004b. "Introduction to this special section on beauty, goodness, and usability." Human-Computer Interaction 19(4):311-318.

Osgood, Charles E, George J Suci and Percy H Tannenbaum. 1957. "The measurement of meaning. Urbana: Univer." of Illinois Press 195:36.

Osgood, Charles E., W. E. May and M. S. Miron. 1975. Cross-Cultural Universals of Affective Meaning. Urbana: University of Illinois. 
Paolacci, Gabriele, Jesse Chandler and Panagiotis Ipeirotis. 2010. "Running experiments on amazon mechanical turk." Judgment and Decision Making 5(5):411-419.

Reed, Americus. 2002. "Social identity as a useful perspective for self-concept-based consumer research." Psychology \& Marketing 19(3):235-266.

Reed, Americus, Mark R. Forehand, Stefano Puntoni and Luk Warlop. 2012. "Identity-based consumer behavior." International Journal of Research in Marketing 29(4):310-321.

Robinson, Dawn T. and Lynn Smith-Lovin. 2006. "Affect Control Theory." in Social Psychology, edited by P. J. Burke. Stanford: Stanford University Press.

Rosenberg, Morris. 1979. Conceiving the Self. New York: Basic Books, Inc.

Schneider, Andreas. 2002. "Behaviour prescriptions versus professional identities in multi-cultural corporations: a cross-cultural computer simulation." Organization Studies 23(1):105-131.

Scholl, Wolfgang. 2013. "The Socio-Emotional Basis of Human Interaction and Communication: How we Construct our Social World." Social Science Information 52(1):3-33.

Shank, Daniel B. 2010. "An Affect Control Theory of Technology." Current Research in Social Psychology 15(10):1-13.

—. 2015. "Using Crowdsourcing Websites for Sociological Research: The Case of Amazon Mechanical Turk." The American Sociologist:1-9.

Sirgy, M. 1982. "Self-concept in consumer behavior: a critical review." Journal of Consumer research:287-300. 
Smith, Herman W, Takanori Matsuno and Shuuichirou Ike. 2001. "The affective basis of attributional processes among Japanese and Americans." Social Psychology Quarterly:180-194.

Solomon, Michael R. 1983. "The role of products as social stimuli: a symbolic interactionism perspective." Journal of Consumer research:319-329.

Stryker, Sheldon. 1980. Symbolic Interactionism: A Social Structural Version. Menlo Park, CA: Benjamin/Cummings.

Stryker, Sheldon and Kevin D. Vyran. 2003. "The Symbolic Interactionism Frame." in Handbook of Social Psychology, edited by J. Delamater. New York: Kluwer.

Thomas, Lisa and David R. Heise. 1995. "Mining Error Variance and Hitting PayDirt: Discovering Systematic Variation in Social Sentiments." The Sociological Quarterly 36:425-439.

Veblen, Thorstein. 2007 [1899]. The theory of the leisure class: Oxford University Press.

Zeithaml, Valarie A. 1988. "Consumer perceptions of price, quality, and value: a means-end model and synthesis of evidence." The Journal of marketing:2-22. 
Table 1: Mean Evaluation, Potency, and Activity of Technology Products by Gender (SD in parentheses)

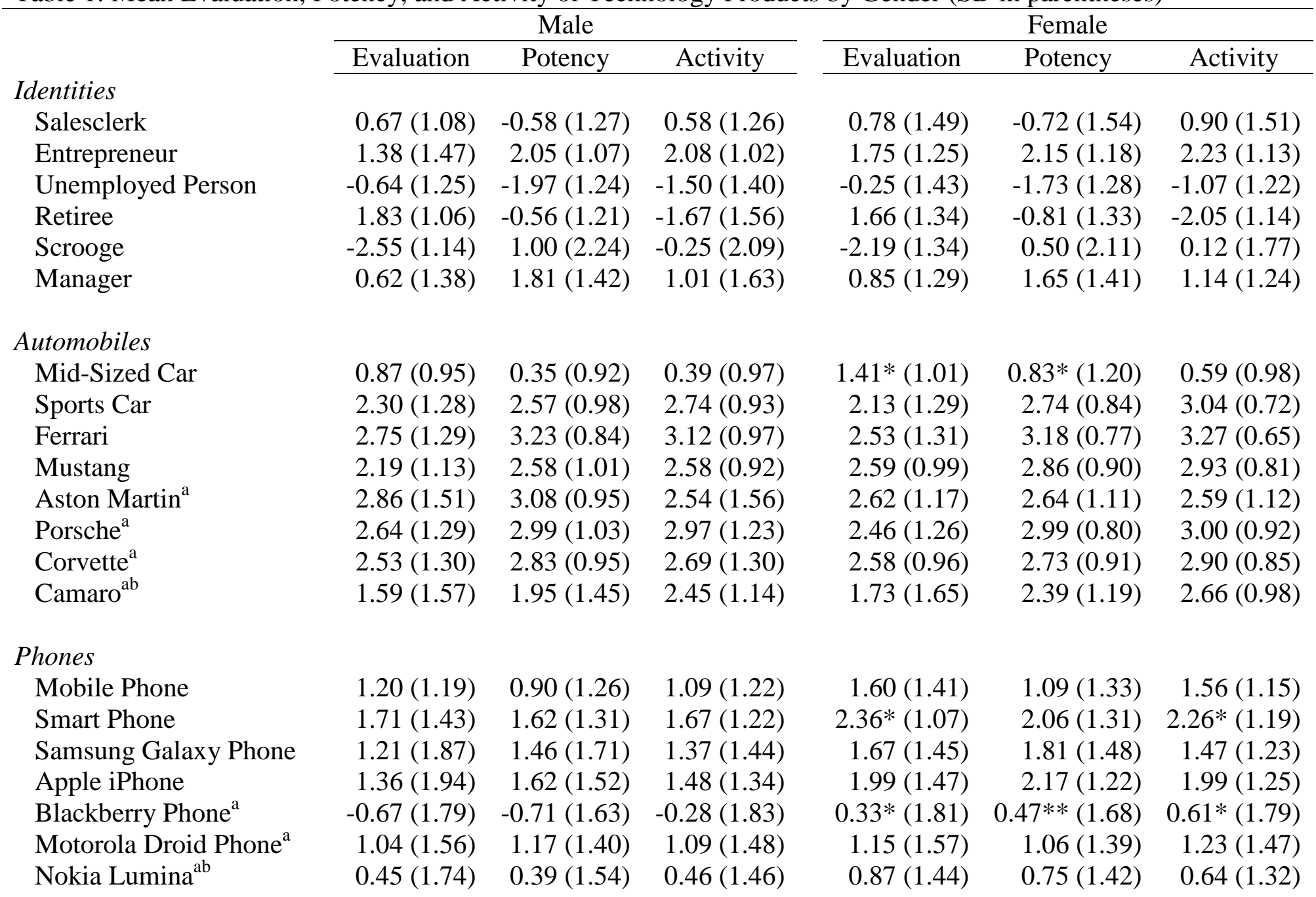


Computers

Laptop

Tablet Computer

$2.07(1.07) \quad 1.59(1.29) \quad 1.42(1.25)$

$1.61(1.60)$

$0.99(1.88) \quad 1.29(1.71)$

$2.09(1.23)$

$1.87(1.30)$

$1.40(1.44)$

Microsoft Surface

$0.96(1.50)$

$0.97(1.48) \quad 0.68(1.49)$

$1.88(1.35) \quad 1.68(1.32)$

$1.25(1.35)$

Apple iPad

$1.62(1.51) \quad 1.44(1.49) \quad 1.52(1.34)$

Amazon Kindle ${ }^{\mathrm{a}}$

$1.46(1.28) \quad 1.04(1.32) \quad 0.41(1.56)$

$2.02(1.27) \quad 2.07 *(1.21) \quad 1.60(1.14)$

Google Nexus $^{\mathrm{a}}$

$1.34(1.35) \quad 1.31(1.31) \quad 1.53(1.03)$

$1.48(1.23)$

$0.32(1.67)$

$1.77(1.42) \quad 1.81(1.36) \quad 1.38(1.36)$

\footnotetext{
${ }^{\mathrm{a}}$ Used for validity comparisons, not for product modification

${ }^{\mathrm{b}}$ Rated in all three sets

$* \mathrm{p} \leq .05, * * \mathrm{p} \leq .01, * * * \mathrm{p} \leq .001$ (gender differences using a two-tailed t-test)
} 
Table 2: Regression Models for Modified Identity’s Evaluation

\begin{tabular}{|c|c|c|c|c|}
\hline & Model 1 & Model 2 & Model 3 & Model 4 \\
\hline Constant & $.248(.023)^{* * *}$ & $.281(.089)^{* *}$ & $.250(.089)^{* *}$ & $.236(.108)^{*}$ \\
\hline \multicolumn{5}{|c|}{ Unmodified Identity } \\
\hline Evaluation & $.556(.016)^{* * *}$ & $.556(.015)^{* * *}$ & $.558(.015)^{* * *}$ & $.558(.015)^{* * *}$ \\
\hline Potency & $.060(.025)^{*}$ & $.061(.024)^{*}$ & $.053(.023)^{*}$ & $.053(.024) *$ \\
\hline Activity & $.037(.026)$ & $.036(.024)$ & $.044(.024) \dagger$ & $.044(.024) \dagger$ \\
\hline \multicolumn{5}{|l|}{ Consumer Product } \\
\hline Evaluation & & $.168(.096) \dagger$ & $.235(.098)^{*}$ & $.191(.136)$ \\
\hline Potency & & $.003(.091)$ & $.045(.091)$ & $.035(.133)$ \\
\hline Activity & & $-.199(.076)^{* *}$ & $-.262(.079)^{* * *}$ & $-.201(.091)^{*}$ \\
\hline Gender & & & $-.110(.046)^{*}$ & $-.107(.047)^{*}$ \\
\hline \multicolumn{5}{|c|}{ Product Characteristics ${ }^{\mathrm{a}}$} \\
\hline Brand & & & & $.018(.062)$ \\
\hline Medium Status & & & & $.027(.070)$ \\
\hline Car & & & & $-.070(.062)$ \\
\hline Computer & & & & $.034(.056)$ \\
\hline $\mathrm{R}^{2}$ & .908 & .921 & .924 & .926 \\
\hline$\Delta \mathrm{R}^{2}$ & $.908 * * *$ & $.013 * * *$ & $.003 *$ & .002 \\
\hline
\end{tabular}

$\mathrm{N}=144$

$+\mathrm{p} \leq .1, * \mathrm{p} \leq .05, * * \mathrm{p} \leq .01, * * * \mathrm{p} \leq .001$

${ }^{a}$ Because there were no branded medium status products the Brand and Medium Status dummy variables both have a reference category of Generic High Status. Phone is the reference category for Car and Computer product type. 
Table 3: Regression Models for Modified Identity’s Potency

\begin{tabular}{|c|c|c|c|c|}
\hline & Model 1 & Model 2 & Model 3 & Model 4 \\
\hline Constant & $.661(.036)^{* * *}$ & $-.069(.095)$ & $-.093(.095)$ & $-.070(.116)$ \\
\hline \multicolumn{5}{|c|}{ Unmodified Identity } \\
\hline Evaluation & $.096(.025)^{* * *}$ & $.093(.016)^{* * *}$ & $.094(.016)^{* * *}$ & $.094(.016)^{* * *}$ \\
\hline Potency & $.359(.039)^{* * *}$ & $.371(.025)^{* * *}$ & $.365(.025)^{* * *}$ & $.365(.025)^{* * *}$ \\
\hline Activity & $.048(.041)$ & $.035(.026)$ & $.041(.026)$ & $.041(.026)$ \\
\hline \multicolumn{5}{|l|}{ Consumer Product } \\
\hline Evaluation & & $-.024(.102)$ & $.028(.105)$ & $.030(.147)$ \\
\hline Potency & & $.413(.096)^{* * *}$ & $.446(.097)^{* * *}$ & $.510(.143)^{* * *}$ \\
\hline Activity & & $.019(.080)$ & $-.031(.084)$ & $-.085(.099)$ \\
\hline Gender & & & $-.086(.049) \dagger$ & $-.098(.051)^{\dagger}$ \\
\hline \multicolumn{5}{|c|}{ Product Characteristics ${ }^{a}$} \\
\hline Brand & & & & $-.041(.067)$ \\
\hline Medium Status & & & & $-.005(.076)$ \\
\hline Car & & & & $-.013(.067)$ \\
\hline Computer & & & & $-.052(.061)$ \\
\hline $\mathrm{R}^{2}$ & .684 & .875 & .878 & .879 \\
\hline $\mathrm{R}^{2} \Delta$ & $.684 * * *$ & $191 * * *$ & $.003 \dagger$ & .001 \\
\hline
\end{tabular}

$\mathrm{N}=144$

$\dagger \mathrm{p} \leq .1, * \mathrm{p} \leq .05, * * \mathrm{p} \leq .01, * * * \mathrm{p} \leq .001$

${ }^{a}$ Because there were no branded medium status products the Brand and Medium Status dummy variables both have a reference category of Generic High Status. Phone is the reference category for Car and Computer product type. 
Table 4: Regression Models for Modified Identity’s Activity

\begin{tabular}{|c|c|c|c|c|}
\hline & Model 1 & Model 2 & Model 3 & Model 4 \\
\hline Constant & $.822(.057)^{* * *}$ & $-.159(.108)$ & $-.229(.102)^{*}$ & $-.310(.115)^{* *}$ \\
\hline \multicolumn{5}{|c|}{ Unmodified Identity } \\
\hline Evaluation & $.033(.039)$ & $.029(.018)$ & $.034(.017)^{*}$ & $.034(.016)^{*}$ \\
\hline Potency & $.042(.063)$ & .055 (.029)† & - $039(.027)$ & .039 (.025) \\
\hline Activity & $.350(.065)^{* * *}$ & $.337(.030)^{* * *}$ & $.354(.028) * * *$ & $.354(.026) * * *$ \\
\hline \multicolumn{5}{|l|}{ Consumer Product } \\
\hline Evaluation & & $-.389(.116)^{* * *}$ & $-.241(.113)^{*}$ & $-.061(.146)$ \\
\hline Potency & & $.424(.11)^{* * *}$ & $.518(.104)^{* * *}$ & $.417(.142)^{* *}$ \\
\hline Activity & & $.536(.092)^{* * *}$ & $.395(.091)^{* * *}$ & $.298(.098)^{* *}$ \\
\hline Gender & & & $-.245(.053)^{* * *}$ & $-.242(.051)^{* * *}$ \\
\hline \multicolumn{5}{|c|}{ Product Characteristics ${ }^{\mathrm{a}}$} \\
\hline Brand & & & & $.079(.066)$ \\
\hline Medium Status & & & & $.005(.075)$ \\
\hline Car & & & & $.238(.066)^{* * *}$ \\
\hline Computer & & & & $-.055(.060)$ \\
\hline $\mathrm{R}^{2}$ & .415 & .882 & .898 & .906 \\
\hline $\mathrm{R}^{2} \Delta$ & $.415^{* * *}$ & $.467 * * *$ & $.016^{* * *}$ & $.016^{* * *}$ \\
\hline
\end{tabular}

$\mathrm{N}=144$

$+\mathrm{p} \leq .1, * \mathrm{p} \leq .05, * * \mathrm{p} \leq .01, * * * \mathrm{p} \leq .001$

${ }^{a}$ Because there were no branded medium status products the Brand and Medium Status dummy variables both have a reference category of Generic High Status. Phone is the reference category for Car and Computer product type. 


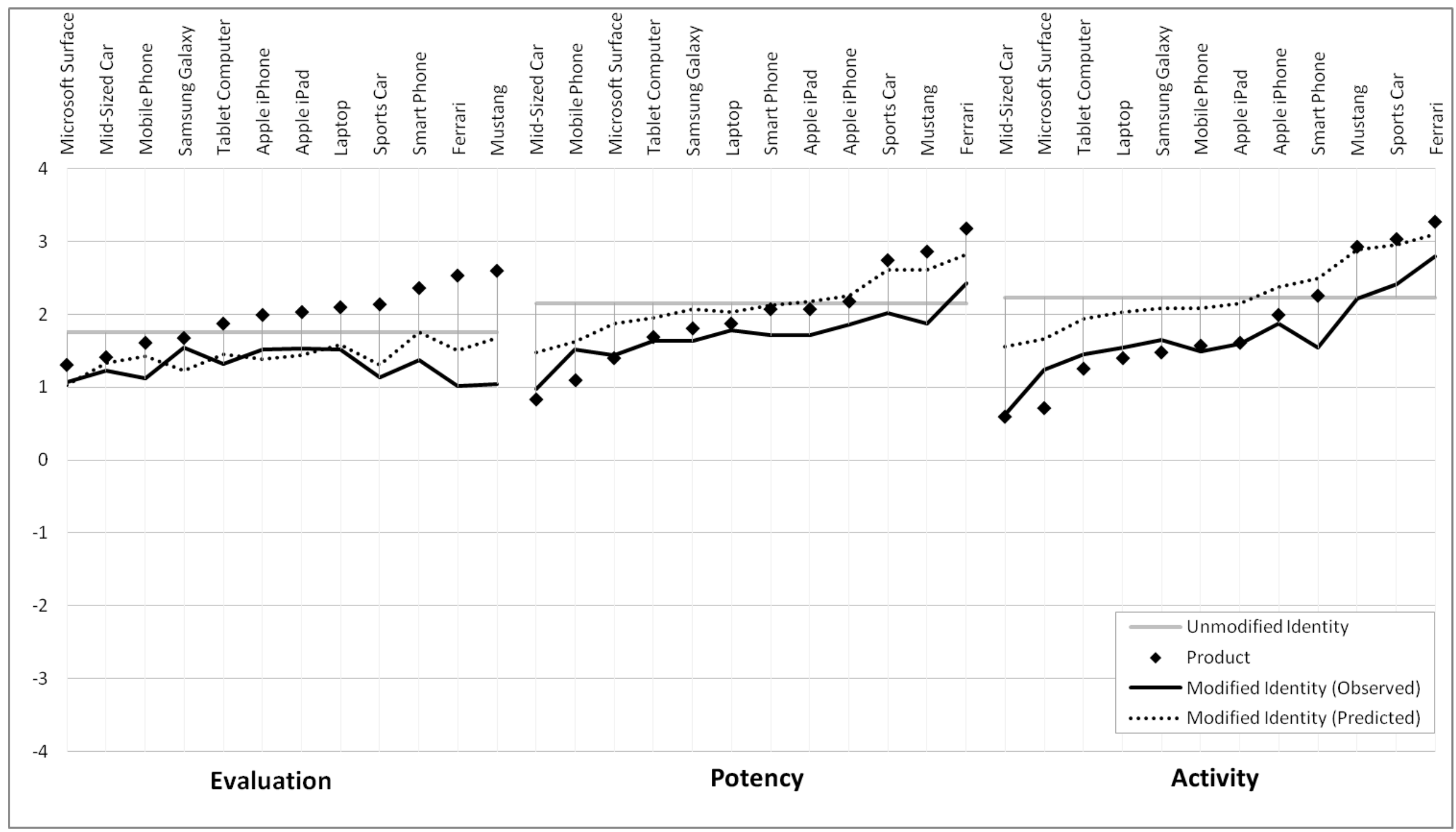

Figure 1: Evaluation, Potency, and Activity for Entrepreneur and products (women only). 


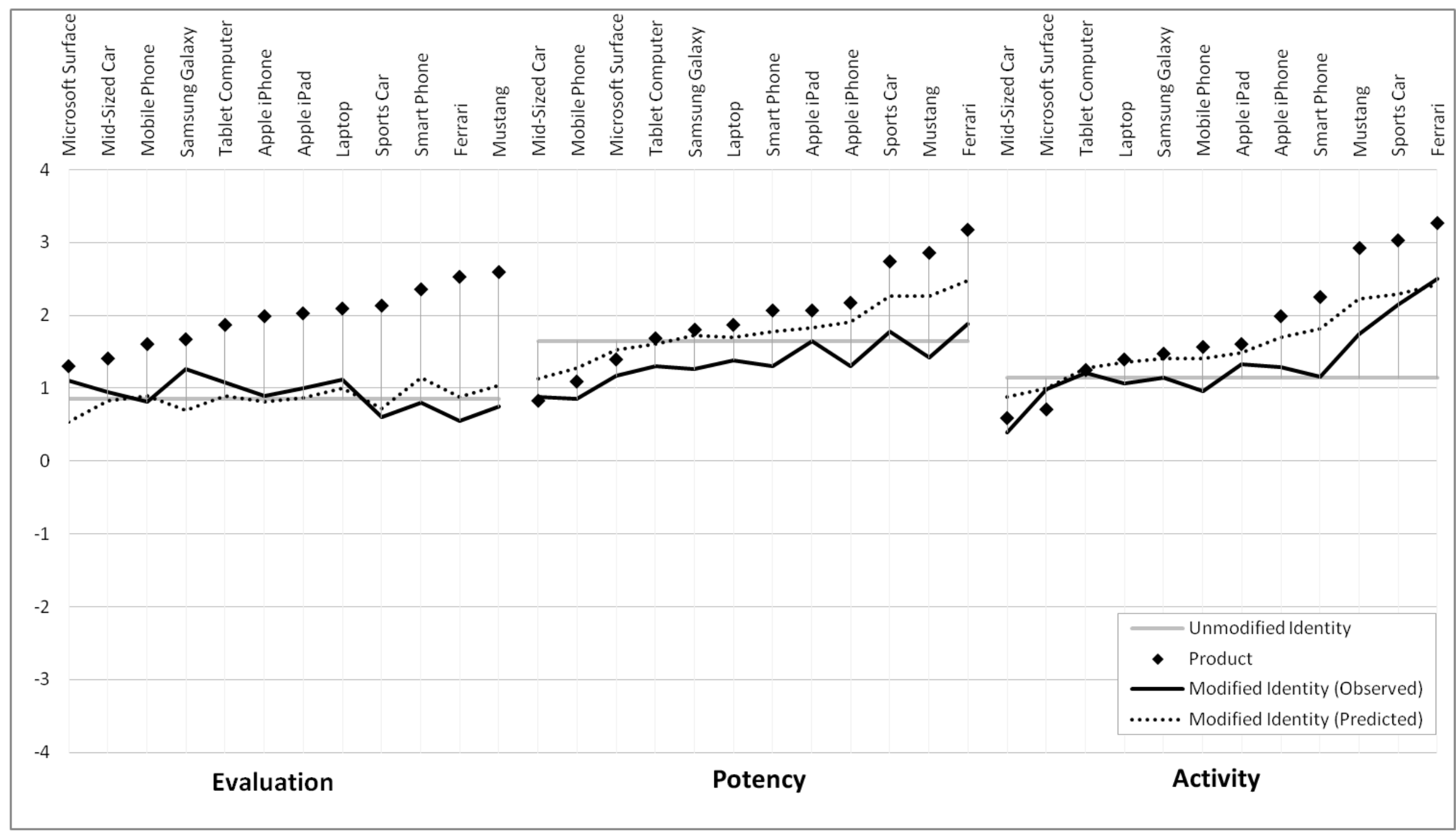

Figure 2: Evaluation, Potency, and Activity for Manager and products (women only). 


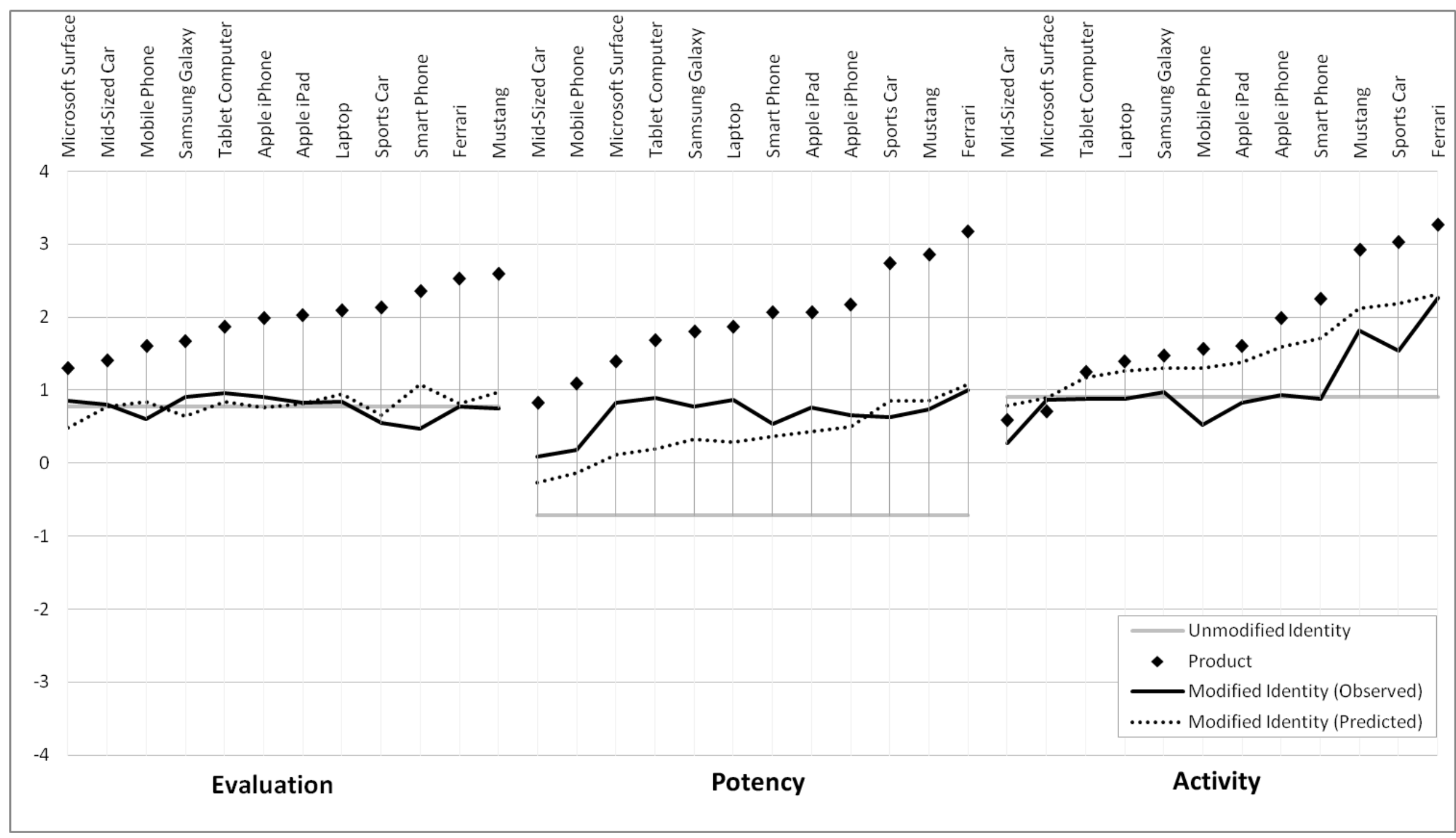

Figure 3: Evaluation, Potency, and Activity for Salesclerk and products (women only). 


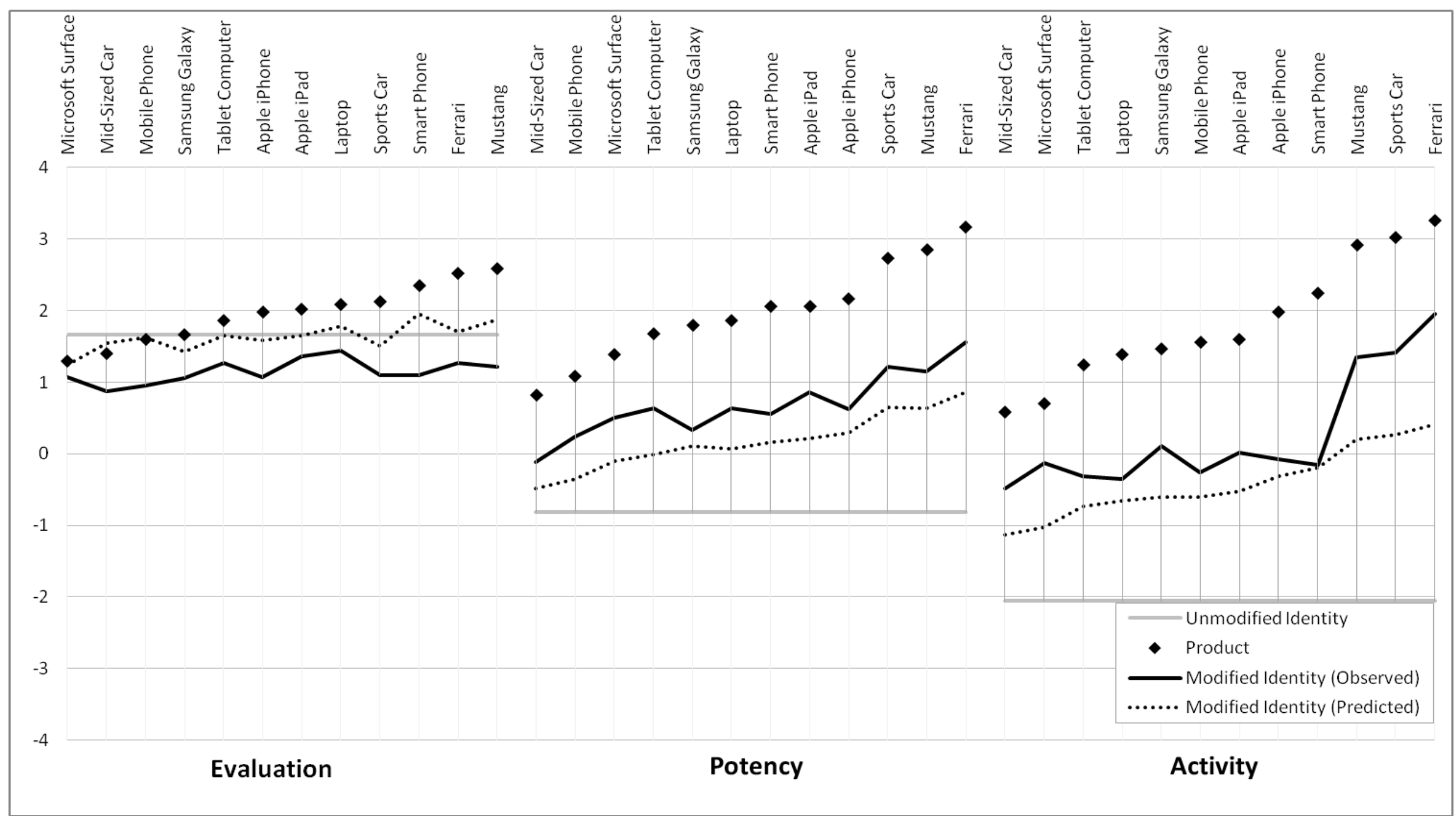

Figure 4: Evaluation, Potency, and Activity for Retiree and products (women only). 


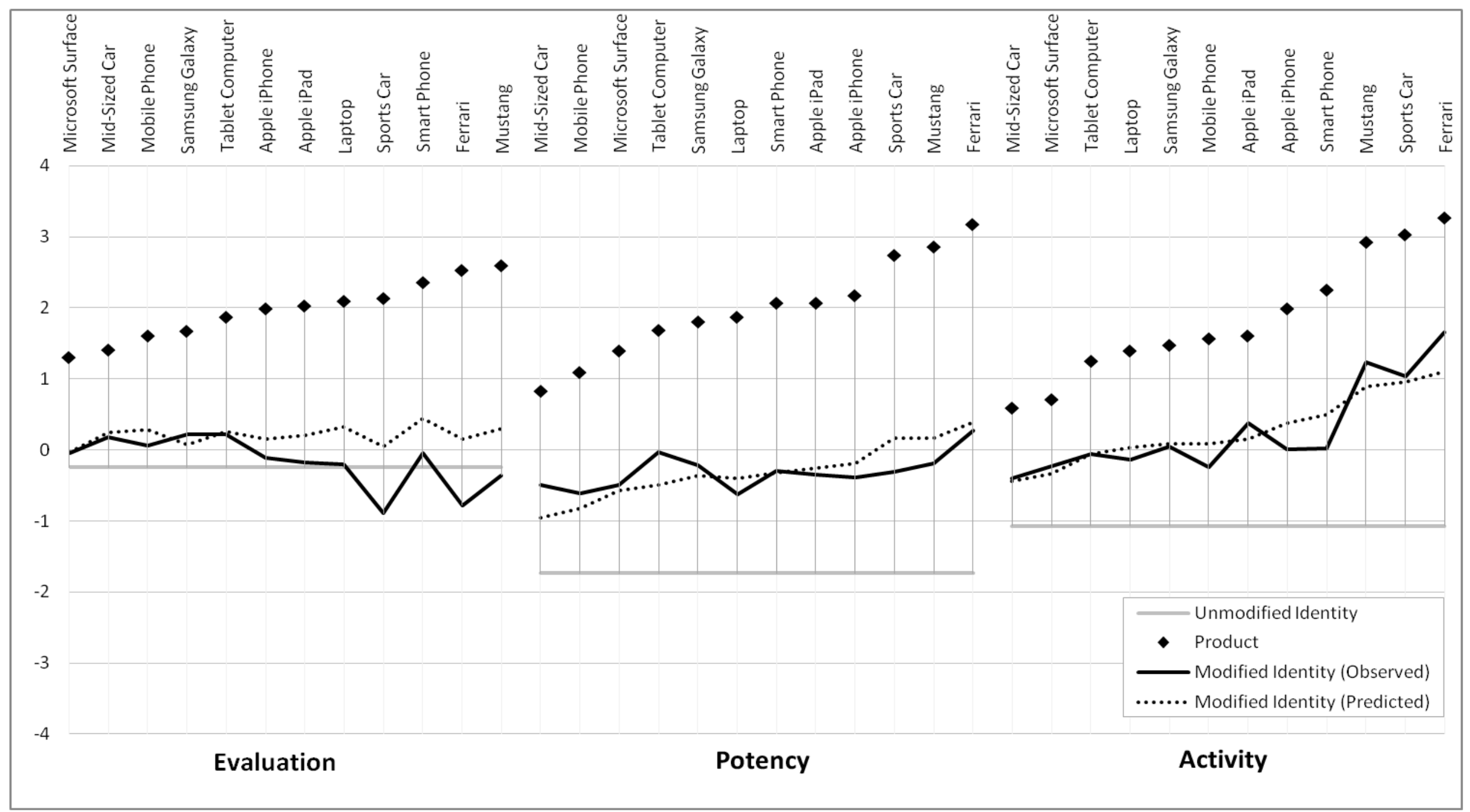

Figure 5: Evaluation, Potency, and Activity for Unemployed and products (women only). 


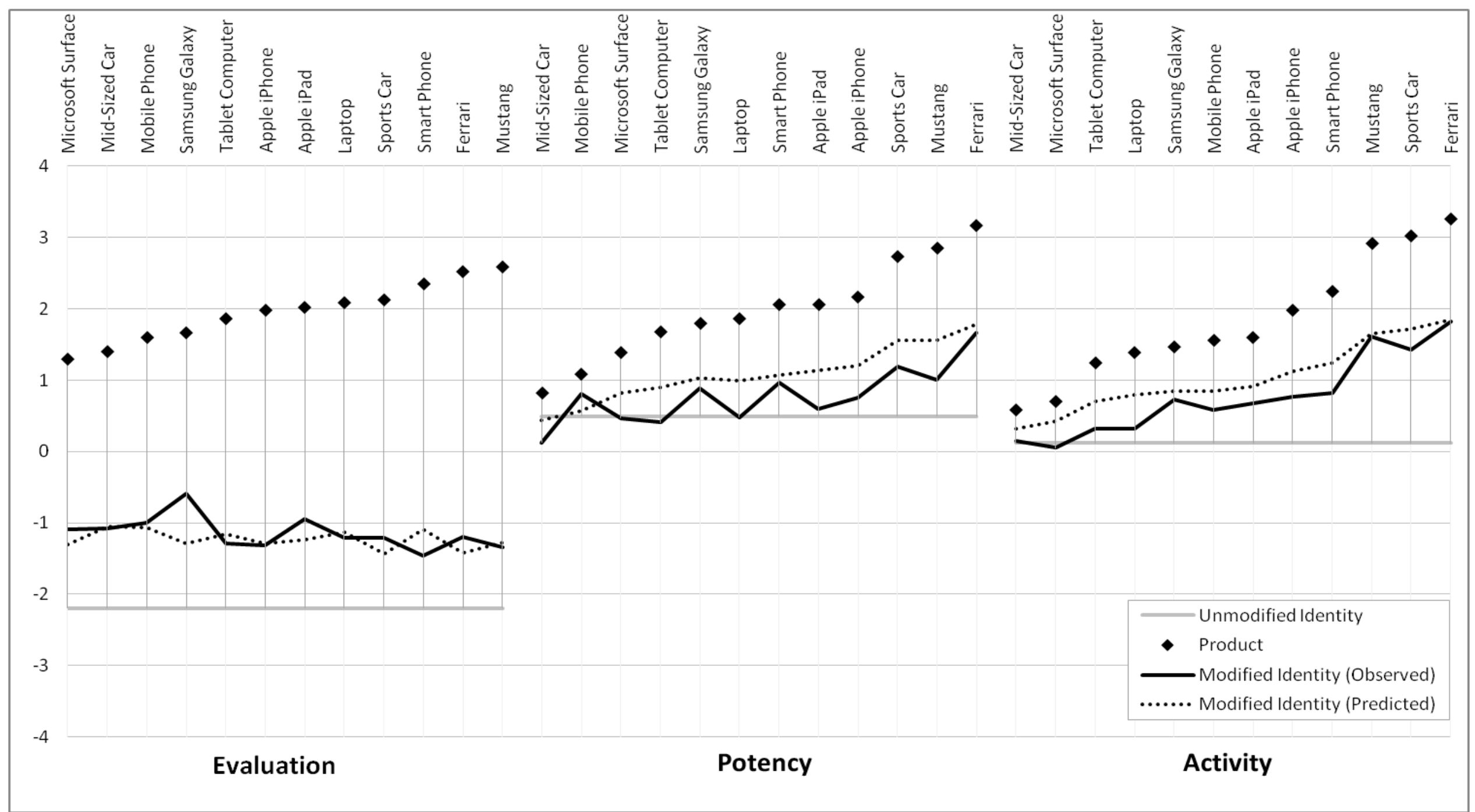

Figure 6: Evaluation, Potency, and Activity for Scrooge and products (women only). 
Appendix Table A: All modified Identity means.

\begin{tabular}{|c|c|c|c|c|c|c|}
\hline & \multicolumn{3}{|c|}{ Male } & \multicolumn{3}{|c|}{ Female } \\
\hline & $\mathrm{E}$ & $\mathrm{P}$ & $\mathrm{A}$ & $\mathrm{E}$ & $\mathrm{P}$ & A \\
\hline Entrepreneur with a Ferrari & 1.37 & 2.52 & 3.01 & 1.01 & 2.43 & 2.80 \\
\hline Entrepreneur with a Laptop & 1.72 & 1.91 & 1.68 & 1.52 & 1.78 & 1.55 \\
\hline Entrepreneur with a Microsoft Surface & 0.86 & 1.26 & 1.26 & 1.07 & 1.43 & 1.23 \\
\hline Entrepreneur with a Mid-Sized Car & 1.02 & 0.76 & 0.68 & 1.22 & 0.97 & 0.62 \\
\hline Entrepreneur with a Mobile Phone & 1.46 & 1.50 & 1.54 & 1.12 & 1.51 & 1.49 \\
\hline Entrepreneur with a Mustang & 0.92 & 1.88 & 2.30 & 1.05 & 1.87 & 2.22 \\
\hline $\begin{array}{l}\text { Entrepreneur with a Samsung Galaxy } \\
\text { Phone }\end{array}$ & 1.35 & 1.58 & 1.75 & 1.55 & 1.63 & 1.65 \\
\hline Entrepreneur with a Smart Phone & 1.32 & 1.64 & 1.70 & 1.37 & 1.71 & 1.54 \\
\hline Entrepreneur with a Sports Car & 1.01 & 1.91 & 2.26 & 1.13 & 2.02 & 2.41 \\
\hline Entrepreneur with a Tablet Computer & 1.18 & 1.34 & 1.50 & 1.32 & 1.63 & 1.45 \\
\hline Entrepreneur with an Apple iPad & 1.10 & 1.02 & 1.22 & 1.53 & $1.72 *$ & 1.59 \\
\hline Entrepreneur with an Apple iPhone & 1.46 & 1.46 & 1.68 & 1.51 & 1.87 & 1.88 \\
\hline Manager with a Ferrari & 0.52 & 2.03 & 2.56 & 0.56 & 1.88 & 2.50 \\
\hline Manager with a Laptop & 0.96 & 1.30 & 0.95 & 1.12 & 1.38 & 1.06 \\
\hline Manager with a Microsoft Surface & 0.71 & 0.98 & 1.09 & 1.10 & 1.17 & 0.99 \\
\hline Manager with a Mid-Sized Car & 0.80 & 0.56 & 0.49 & 0.95 & 0.87 & 0.40 \\
\hline Manager with a Mobile Phone & 0.61 & 0.90 & 0.88 & 0.81 & 0.85 & 0.96 \\
\hline Manager with a Mustang & 0.69 & 1.36 & 1.84 & 0.74 & 1.42 & 1.74 \\
\hline Manager with a Samsung Galaxy Phone & 0.66 & 1.13 & 0.91 & $1.27^{*}$ & 1.26 & 1.15 \\
\hline Manager with a Smart Phone & 0.96 & 0.96 & 1.17 & 0.80 & 1.30 & 1.15 \\
\hline Manager with a Sports Car & 0.68 & 1.84 & 2.24 & 0.60 & 1.78 & 2.15 \\
\hline Manager with a Tablet Computer & 0.97 & 1.33 & 1.34 & 1.08 & 1.31 & 1.21 \\
\hline Manager with an Apple iPad & 1.17 & 1.47 & 1.39 & 1.01 & 1.64 & 1.33 \\
\hline Manager with an Apple iPhone & 0.64 & 0.81 & 0.95 & 0.89 & 1.30 & 1.29 \\
\hline Retiree with a Ferrari & 1.41 & 1.52 & 1.82 & 1.27 & 1.56 & 1.96 \\
\hline Retiree with a Laptop & 1.20 & 0.02 & -0.41 & 1.44 & $0.64 *$ & -0.35 \\
\hline Retiree with a Microsoft Surface & 1.10 & 0.27 & -0.31 & 1.08 & 0.51 & -0.13 \\
\hline Retiree with a Mid-Sized Car & 0.98 & 0.21 & -0.29 & 0.87 & -0.11 & -0.49 \\
\hline Retiree with a Mobile Phone & 0.94 & 0.03 & -0.43 & 0.96 & 0.24 & -0.25 \\
\hline Retiree with a Mustang & 1.12 & 1.24 & 1.18 & 1.22 & 1.16 & 1.35 \\
\hline Retiree with a Samsung Galaxy Phone & 1.14 & 0.48 & -0.15 & 1.06 & 0.33 & 0.11 \\
\hline Retiree with a Smart Phone & 1.09 & 0.61 & -0.11 & 1.10 & 0.56 & -0.15 \\
\hline Retiree with a Sports Car & 1.19 & 1.14 & 1.31 & 1.10 & 1.22 & 1.42 \\
\hline Retiree with a Tablet Computer & 1.00 & 0.25 & -0.45 & 1.27 & 0.64 & -0.31 \\
\hline Retiree with an Apple iPad & 0.74 & 0.09 & -0.69 & 1.36 & $0.86^{*}$ & 0.01 \\
\hline Retiree with an Apple iPhone & 0.77 & -0.02 & -0.22 & 1.07 & $0.62 *$ & -0.08 \\
\hline Salesclerk with a Ferrari & 0.70 & 1.51 & 2.38 & 0.77 & 1.00 & 2.27 \\
\hline Salesclerk with a Laptop & 0.89 & 0.59 & 0.75 & 0.85 & 0.86 & 0.89 \\
\hline Salesclerk with a Microsoft Surface & 0.48 & 0.36 & 0.45 & 0.85 & 0.83 & 0.87 \\
\hline Salesclerk with a Mid-Sized Car & 0.60 & 0.37 & 0.40 & 0.80 & 0.10 & 0.27 \\
\hline
\end{tabular}




\begin{tabular}{|c|c|c|c|c|c|c|}
\hline Salesclerk with a Mobile Phone & 0.57 & 0.56 & 0.95 & 0.61 & 0.19 & 0.52 \\
\hline Salesclerk with a Mustang & 0.52 & 1.19 & 1.76 & 0.75 & 0.74 & 1.82 \\
\hline $\begin{array}{l}\text { Salesclerk with a Samsung Galaxy } \\
\text { Phone }\end{array}$ & 1.00 & 0.79 & 0.96 & 0.91 & 0.77 & 0.97 \\
\hline Salesclerk with a Smart Phone & 0.78 & 0.57 & 0.99 & 0.48 & 0.54 & 0.89 \\
\hline Salesclerk with a Sports Car & 0.67 & 1.30 & 1.81 & 0.55 & $0.63 *$ & 1.54 \\
\hline Salesclerk with a Tablet Computer & 0.83 & 0.43 & 1.20 & 0.96 & 0.89 & 0.88 \\
\hline Salesclerk with an Apple iPad & 0.76 & 0.40 & 1.15 & 0.83 & 0.76 & 0.83 \\
\hline Salesclerk with an Apple iPhone & 0.83 & 0.80 & 1.13 & 0.91 & 0.66 & 0.93 \\
\hline Scrooge with a Ferrari & -1.13 & 1.58 & 2.16 & -1.20 & 1.66 & 1.82 \\
\hline Scrooge with a Laptop & -0.90 & 0.74 & 0.62 & -1.21 & 0.48 & 0.32 \\
\hline Scrooge with a Microsoft Surface & -0.87 & 0.46 & 0.56 & -1.10 & 0.47 & 0.05 \\
\hline Scrooge with a Mid-Sized Car & -0.77 & 0.40 & -0.04 & -1.08 & 0.12 & 0.15 \\
\hline Scrooge with a Mobile Phone & -1.15 & 0.61 & 0.13 & -1.00 & 0.80 & 0.59 \\
\hline Scrooge with a Mustang & -0.97 & 0.96 & 1.78 & -1.34 & 1.01 & 1.61 \\
\hline Scrooge with a Samsung Galaxy Phone & -1.09 & 0.73 & 0.23 & -0.60 & 0.88 & 0.72 \\
\hline Scrooge with a Smart Phone & -1.33 & 0.70 & 0.17 & -1.46 & 0.97 & 0.82 \\
\hline Scrooge with a Sports Car & -0.88 & 1.20 & 1.51 & -1.22 & 1.19 & 1.43 \\
\hline Scrooge with a Tablet Computer & -0.71 & 0.79 & 0.28 & -1.29 & 0.41 & 0.32 \\
\hline Scrooge with an Apple iPad & -0.79 & 0.81 & 0.44 & -0.94 & 0.60 & 0.68 \\
\hline Scrooge with an Apple iPhone & -0.79 & 0.89 & 0.48 & -1.31 & 0.76 & 0.77 \\
\hline Unemployed Person with a Ferrari & -0.93 & 0.70 & 2.15 & -0.78 & 0.27 & 1.66 \\
\hline Unemployed Person with a Laptop & 0.15 & -0.33 & -0.28 & -0.20 & -0.63 & -0.14 \\
\hline $\begin{array}{l}\text { Unemployed Person with a Microsoft } \\
\text { Surface }\end{array}$ & -0.35 & -0.77 & -0.54 & -0.05 & -0.49 & -0.24 \\
\hline $\begin{array}{l}\text { Unemployed Person with a Mid-Sized } \\
\text { Car }\end{array}$ & 0.45 & -0.57 & -0.43 & 0.18 & -0.50 & -0.40 \\
\hline $\begin{array}{l}\text { Unemployed Person with a Mobile } \\
\text { Phone }\end{array}$ & -0.03 & -0.75 & -0.22 & 0.06 & -0.62 & -0.25 \\
\hline Unemployed Person with a Mustang & -0.82 & -0.10 & 1.08 & -0.36 & -0.19 & 1.24 \\
\hline $\begin{array}{l}\text { Unemployed Person with a Samsung } \\
\text { Galaxy Phone }\end{array}$ & 0.00 & -0.17 & 0.06 & 0.22 & -0.21 & 0.05 \\
\hline $\begin{array}{l}\text { Unemployed Person with a Smart } \\
\text { Phone }\end{array}$ & -0.17 & -0.50 & -0.23 & -0.05 & -0.30 & 0.02 \\
\hline Unemployed Person with a Sports Car & -0.71 & -0.18 & 1.41 & -0.89 & -0.30 & 1.04 \\
\hline $\begin{array}{l}\text { Unemployed Person with a Tablet } \\
\text { Computer }\end{array}$ & -0.18 & -0.55 & -0.14 & 0.22 & -0.03 & -0.06 \\
\hline Unemployed Person with an Apple iPad & -0.13 & -0.32 & 0.48 & -0.18 & -0.35 & 0.38 \\
\hline $\begin{array}{l}\text { Unemployed Person with an Apple } \\
\text { iPhone }\end{array}$ & -0.63 & -0.49 & -0.14 & -0.11 & -0.39 & 0.01 \\
\hline
\end{tabular}


${ }^{1}$ Values are from a male perspective using the Indiana 2004 ACT dictionary.

${ }^{2}$ The six identities chosen were all categorized as identities related to the domain of business in the affect control theory dictionaries, and were selected because they represented diverse areas in EPA space as follows (- for negative, 0 for near neutral, and + for positive values): salesclerk ( 00 0), entrepreneur $(+++)$, unemployed person (- - -), retiree $(+0$-), scrooge $(-00)$, manager $(0++)$. Values shown in text are from the Indiana 2004 ACT dictionary (Francis and Heise 2006) and averaged across genders.

${ }^{3}$ Unemployed Person received an evaluation of -1.57 for men and -1.40 for women in the Indiana 2004 ACT dictionary (Francis and Heise 2006).

${ }^{4}$ We considered including interaction effects of gender with consumer products and with unmodified identities, but they produced extremely high multicollinearity (VIFs $>100$ ) which would have biased the models.

${ }^{5} \mathrm{R}^{2}$ can be computed by squaring the Pearson correlation of the observed and predicted or by regressing the observed on the predicted. 